\title{
p38ß, A Novel Regulatory Target of Pokemon in Hepatic Cells
}

\author{
Zhe Chen ${ }^{1,2}$, Feng Liu ${ }^{2, *}$, Nannan Zhang ${ }^{2}$, Deliang Cao ${ }^{3}$, Min Liu ${ }^{2}$, Ying Tan ${ }^{2}$ \\ and Yuyang Jiang $1,2,4, *$
}

1 Department of Chemistry, Tsinghua University, Beijing 100084, China;

E-Mail: czseasky@163.com

2 The Ministry-Province Jointly Constructed Base for State Key Lab-Shenzhen Key Laboratory of Chemical Biology, Graduate School at Shenzhen, Tsinghua University, Shenzhen 518055, China;

E-Mails: xiaoee2728@126.com (N.Z.); liumin880608@163.com (M.L.); tan.ying@sz.tsinghua.edu.cn (Y.T.)

3 Department of Medical Microbiology, Immunology \& Cell Biology, Simmons Cooper Cancer Institute, Southern Illinois University School of Medicine, Springfield, IL 62702, USA;

E-Mail: dcao@siumed.edu

4 Department of Pharmacology and Pharmaceutical Sciences, School of Medicine, Tsinghua University, Beijing 100084, China

* Authors to whom correspondence should be addressed;

E-Mails: liu.feng@sz.tsinghua.edu.cn (F.L.); jiangyy@sz.tsinghua.edu.cn (Y.J.);

Tel.: +86-755-2603-6035 (F.L.); Fax: +86-755-2603-2094 (F.L.);

Tel./Fax: +86-755-2603-6017 (Y.J.).

Received: 2 May 2013; in revised form: 8 June 2013 / Accepted: 10 June 2013 /

Published: 27 June 2013

\begin{abstract}
Pokemon is an important proto-oncogene involved in various biological processes and cancer development, such as cell differentiation, tumorigenesis and metastasis. Pokemon is recognized as a transcription factor localized upstream of several oncogenes, regulating their expression. p38MAPKs act as key regulatory factors in cellular signaling pathways associated with inflammatory responses, cell proliferation, differentiation and survival. p38 $\beta$, a member of p38MAPK family, is closely correlated with tumorigenesis, but the mechanism of activation remains unclear. In this study, we found overexpression of Pokemon promoted the growth, migration and invasion of HepG2 cells. However, a p38 inhibitor SB202190 efficiently attenuated the promoting effect of Pokemon in the HepG2 cells. Targeted expression or silencing of Pokemon changed cellular p38 $\beta$ protein level and phosphorylation of downstream ATF2 in the p38 signaling
\end{abstract}


pathway. Both dual luciferase report assay and ChIP assay suggested that $\mathrm{p} 38 \beta$ is a novel regulatory target of the transcription factor Pokemon and positively regulated by Pokemon in hepatic cells.

Keywords: Pokemon; p38ß; transcription factor; hepatic cell; SB202190

\section{Introduction}

Pokemon (POK erythroid myeloid ontogenic factor), also named as Zbtb7A, LRF, FBI-1 and OCZF, contains an $N$-terminal POZ/BTB domain mediating protein-protein interaction and a $C$-terminal Krüppel zinc finger domain responsible for DNA binding [1]. Pokemon is an important transcriptional factor with multiple biological functions, which was originally identified by specifically binding to the inducer of short transcripts (IST) element of human wild type HIV-1 [1]. Pokemon is essential for erythroid differentiation and maturation [2] and fate decision of $\mathrm{B}$ versus $\mathrm{T}$ lymphoid lineage [3]. Pokemon interacts with POZ family members to form a homo- or hetero-dimer participating in various biological processes through its POZ/BTB domain [4]. Pokemon-deficient mouse embryonic fibroblasts (MEFs) are completely unresponsive to either proliferative stimuli or transformative signals of oncogene combinations [5,6].

Pokemon is overexpressed in multiple carcinomas [7-10] and triggers abnormal expression of effector genes and related signaling [11]. Pokemon acts as a transactive or suppressive transcription factor [12,13], inhibiting p53 (mediated through the ARF-Mdm2-p53 pathway) [5] and Rb gene transcription [14] but promoting NF- $\kappa B$ transcription and nuclear localization $[15,16]$. Therefore, Pokemon plays a pivotal role in tumorigenesis as a proto-oncogene localized upstream of several oncogenes [17].

p38MAPKs (p38 mitogen-activated protein kinases) are MAPK family members and key regulatory factors in cellular signaling pathways involved in inflammatory response and cell proliferation, differentiation, survival, migration and invasion [18-21]. Consistent with the importance of these events in tumorigenesis, p38MAPK signaling is associated with human cancers. p38MAPKs are phosphorylation-activated by environmental stresses and mitogens, such as UV irradiation, inflammatory cytokines and growth factors [22], followed by activation of a series of signaling proteins in the pathways and leading to relative biological events. Among the downstream proteins, MSK1, MAPKAPK2 and ATF2 are direct substrates of p38 [23-25].

p38MAPKs consist of 4 members: $338 \alpha, p 38 \beta, p 38 \gamma$ and $\mathrm{p} 38 \delta$, which are encoded by four different genes. p38 $\alpha$ and p38 $\beta$ are ubiquitously expressed in most cell types, while p38 $\gamma$ and p38 $\delta$ have more restricted expression patterns and specialized functions [23]. p38 $\beta$ encoded by the MAPK11 gene shows the highest sequence identity to p38 , but they share distinct substrate affinity and biological functions [26]; their signaling regulation is different [27,28]. p38 $\beta$ emerges at low levels in most cells and tissues, and its contribution to tumorigenesis is not fully clear [29,30].

In this study, we found that a p38 inhibitor SB202190 efficiently attenuated the promoting effect of Pokemon on cell growth, migration and invasion in the HepG2 cells. Consequently, we specifically targeted Pokemon expression in different hepatic cell lines and estimated the effect on the expressions 
of $\mathrm{p} 38 \alpha, \mathrm{p} 38 \beta$ and phosphorylated downstream signaling proteins ATF2. Finally, we identified p38 $\beta$ as a new regulatory target of Pokemon in hepatic cells, which is perhaps a new mechanism through which Pokemon is involved in different biological processes.

\section{Results and Discussion}

\subsection{Pokemon Promotes HepG2 Cell Growth, Migration and Invasion}

We examined Pokemon expression in several hepatic cell lines and found the expression level of Pokemon is correlated with the malignancy of hepatic cells (Figure 1). The protein level of Pokemon is high in malignant hepatocarcinoma cells BEL7404, but low in immortalized hepatic cells HL7702. HepG2 cells were derived from a hepatoblastoma, but are not tumorigenic in nude mice, in which Pokemon is expressed at a moderate level.

Figure 1. Endogenous Pokemon expression in different hepatic cell lines. (a) Western blot in which cells were lysed and total proteins were collected in HepG2, BEL7404, QGY7703 and HL7702 cells, respectively; (b) Quantification of western blot data.

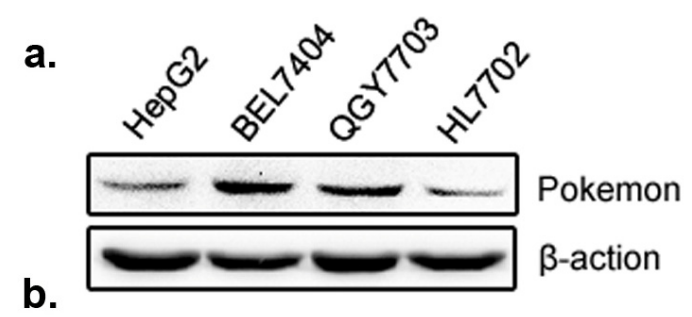

b.

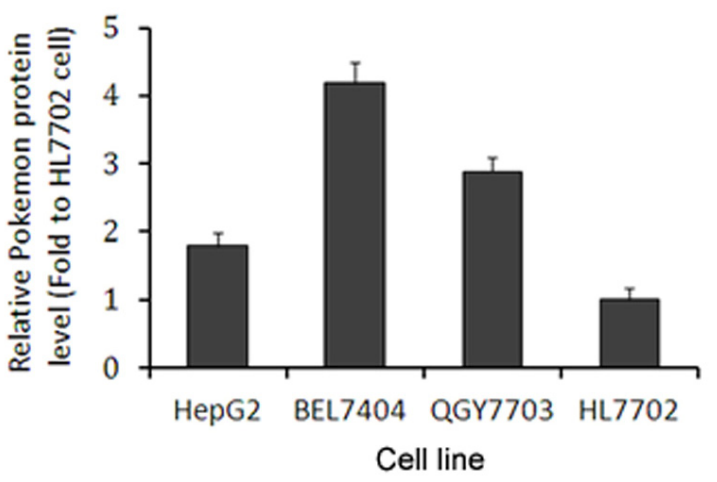

It is reported that Pokemon acts as an oncogene and plays a critical role in tumorigenesis [5]. Hence, we wondered what were the biological effects of Pokemon in hepatic cells. For better comparison, we chose HepG2 cells with a moderate endogenous Pokemon level for ectopic expression or silencing manipulation, and cell growth was estimated by MTT assays. Our results showed that ectopic expression of Pokemon significantly promoted HepG2 proliferation (Figure 2a(i)); however, Pokemon silencing did not notably affect HepG2 cell proliferation (Figure 2a(ii)). To further confirm this finding, we up-regulated Pokemon expression in immortalized hepatic cells HL7702 and silenced Pokemon in hepatocarcinoma cells BEL7404, and obtained consistent results (Figure S1).

We further examined the effect of Pokemon on clonogenic growth in HepG2 cells and the results showed Pokemon overexpression notably enhanced the number of colony formations (Figure 2b(i)), and inversely, the silencing of Pokemon significantly inhibited colony formation (Figure 2b(ii)). 
Because of population dependence, the growth behavior of single cells in colony formation is extremely different from the proliferation of massive cells in the MTT assay.

A main characteristic of malignant cells is the invasive and metastatic ability [31]. To understand the role of Pokemon in HepG2 cell metastasis, we conducted transwell migration and invasion assays. The results demonstrated that Pokemon overexpression significantly promoted the migration (Figure 2c(i)) and invasion (Figure 2d(i)) of HepG2 cells while silencing by si-RNA reduced the migration (Figure 2c(ii)) and invasion (Figure 2d(ii)), and consistent results were obtained with using BEL7404 cells with silenced Pokemon (Figure S2).

Figure 2. Pokemon promotes HepG2 cell growth and metastasis. (a) HepG2 cell growth rate: i. Cells were transfected with pcDNA3.1(-)-Pokemon (marked as Pok) or pcDNA3.1(-) (marked as 3.1); ii. Pokemon was silenced by si-RNA (marked as si-Pok) or scramble RNA (marked as N.C.) in HepG2 cells; (b) Effect of Pokemon on colony formation in HepG2 cells: i. Cells were transfected with pcDNA3.1(-)-Pokemon or pcDNA3.1(-); ii. Pokemon was silenced by si-RNA or scramble RNA in HepG2 cells, Bar chart below the photo stands for the colony formation rate $(\%)$. The colony formation rate stands for the proportion of final clone number accounted for in plated cell number; (c) In vitro migration assays: Pokemon was overexpressed (i) or silenced (ii) in HepG2 cells; (d) In vitro invasion assays. Pokemon was overexpressed (i) or silenced (ii) in HepG2 cells. Bar chart below the photo stands for the relative fold of the migrated or invaded cell number compared to the negative control group. ${ }^{*} p<0.05$ compared to the negative control group.

a.

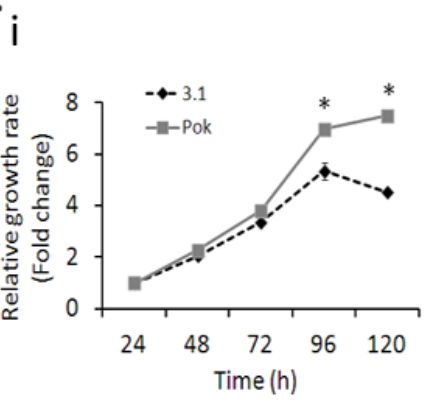

c.
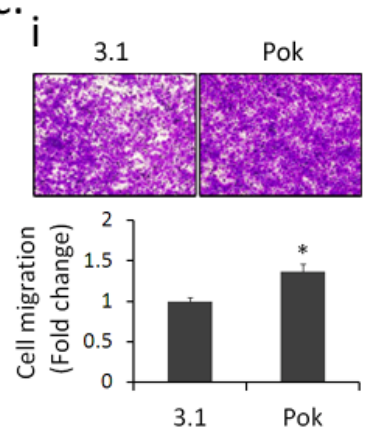

ii

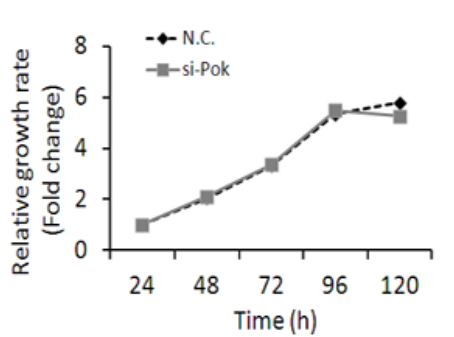

d.
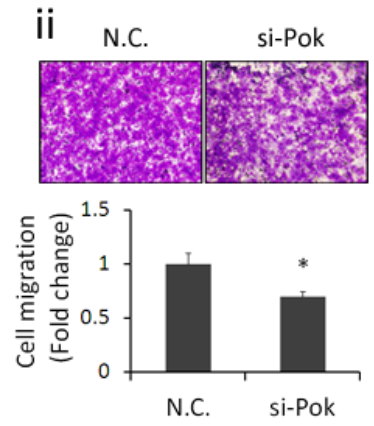

b.
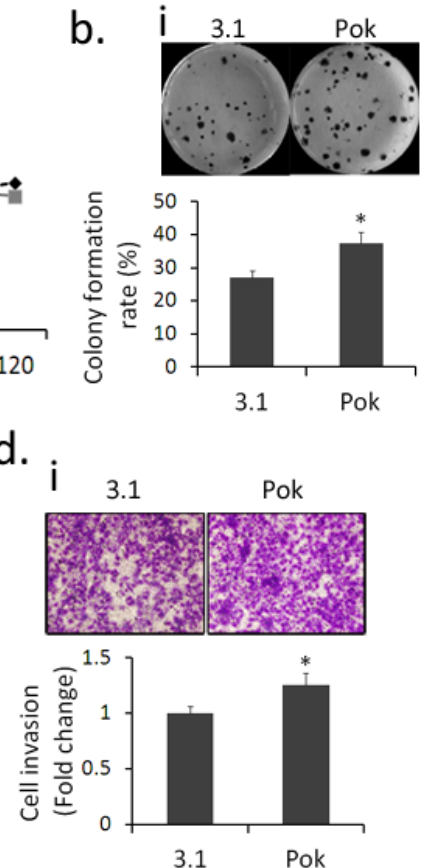

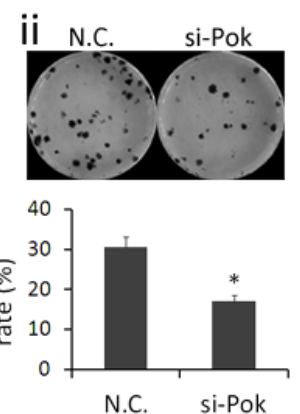

ii N.C. si-Pok
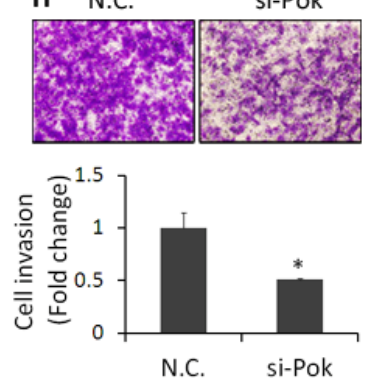


\section{2. p38 Inhibitor SB202190 Attenuates the Promotion of Pokemon on HepG2 Growth, Migration and Invasion}

p38 inhibitor SB202190 specifically inhibits the activity of p38 $\beta$ and $\mathrm{p} 38 \alpha$ [32]. We found SB202190 had dose-dependent inhibition on the proliferation of HL7702, HepG2 and BEL7404 cells, and the inhibition on hepatocarcinoma cells BEL7404 and hepatoblastoma-derived cells HepG2 was more significant (Figure 3a). SB202190 inhibited the phosphorylation of p38 downstream proteins MAPKAPK2, ATF2, MSK1 and HSP27 in a dose-dependent manner, and the inhibition was notable at the concentration of $25 \mu \mathrm{M}$. However, SB202190 slightly increased the phosphorylation of p38, a result similar to that reported using its analogue, SB203580 [24], and it had no significant effect on Pokemon expression (Figure 3b).

We further found that SB202190 $(25 \mu \mathrm{M})$ suppressed the stimulation of ectopic Pokemon on HepG2 proliferation (Figure 3c) and colony formation (Figure 3d). Similarly, SB202190 (25 $\mu$ M) efficiently attenuated the stimulative role of Pokemon in cell migration (Figure 3e) and invasion (Figure 3f). Moreover, we further found Pokemon overexpression increased the phosphorylation of p38 downstream proteins in HepG2 cells, and $25 \mu \mathrm{M}$ SB202190 effectively inhibited the phosphorylation levels with no effect on Pokemon expression (Figure 3g), which indicated that the effect of Pokemon on HepG2 cell growth and metastasis may be associated with p38 pathway.

Figure 3. (a) Inhibition of SB202190 on cell viability: MTT assays in HepG2, BEL7404 and HL7702 cells treated with SB202190 for $48 \mathrm{~h}$ at different concentrations $(0,2.5,5,10$, 25 and $50 \mu \mathrm{M}$ ); (b) Western blot: Displaying that SB202190 dose-dependently inhibits the phosphorylation of p38 downstream proteins. HepG2 cells were treated with SB202190 for $24 \mathrm{~h}$ at different concentrations $(0,10,25$ and $50 \mu \mathrm{M}) ;(\mathbf{c}-\mathbf{g})$ HepG2 cells were treated with $25 \mu \mathrm{M} \mathrm{SB} 202190$ at $24 \mathrm{~h}$ after transfecting with pcDNA3.1(-)-Pokemon or pcDNA3.1(-): (c) HepG2 Cell growth rate; (d) Effect of Pokemon and p38 inhibitor SB202190 on colony formation in HepG2 cells, the colony formation rate stands for the proportion of final clone number accounted for in plated cell number; (e) In vitro migration assays; (f) In vitro invasion assays. Bar chart below the photo stands for the relative fold of the migrated or invaded cell number compared to the negative control group; (g) Pokemon activates p38 signaling pathway in hepatic cells: Left panel is Western blot bands. Western blot in HepG2 cells after Pokemon was overexpressed for $60 \mathrm{~h}$, and the cells were treated by SB202190 at the concentration of $25 \mu \mathrm{M}$; right panel is quantification of western blot data. $* p<0.05$ compared to the negative control group.

a.

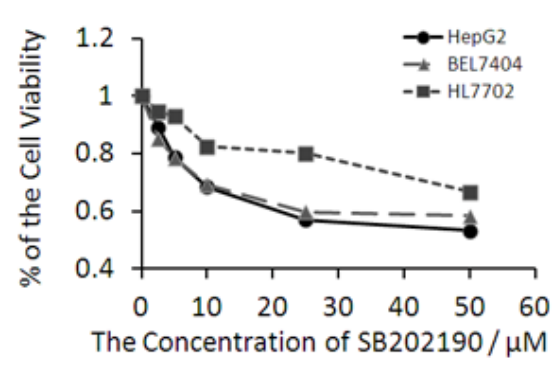

b.

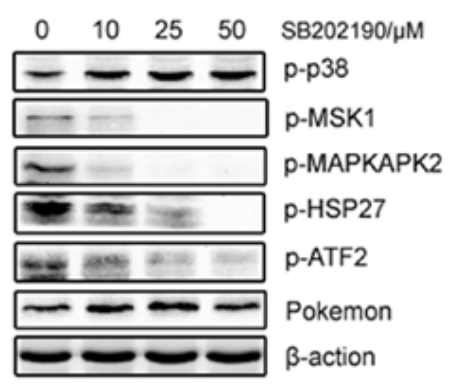

C.

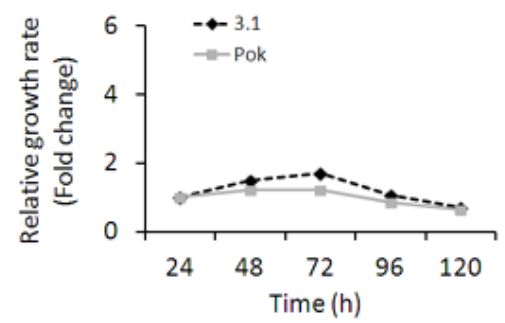


Figure 3. Cont.

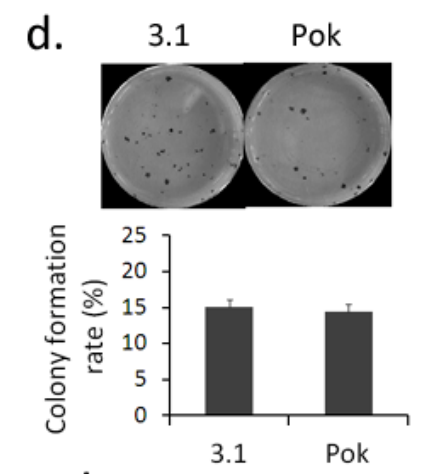

g. i

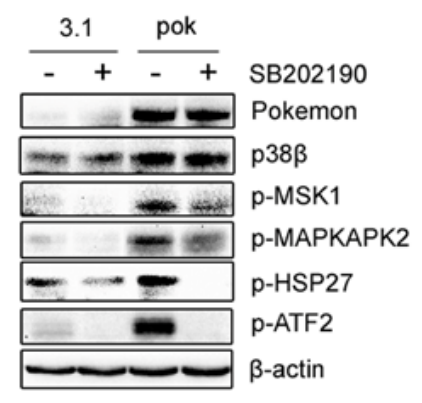

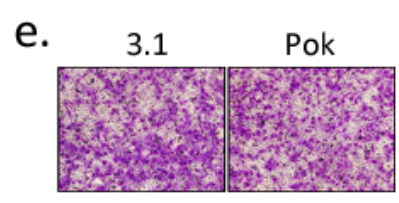

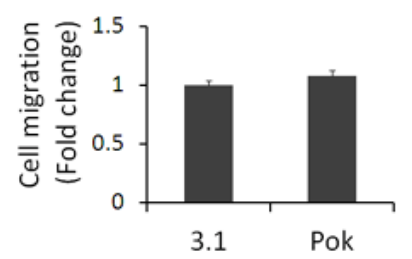

ii

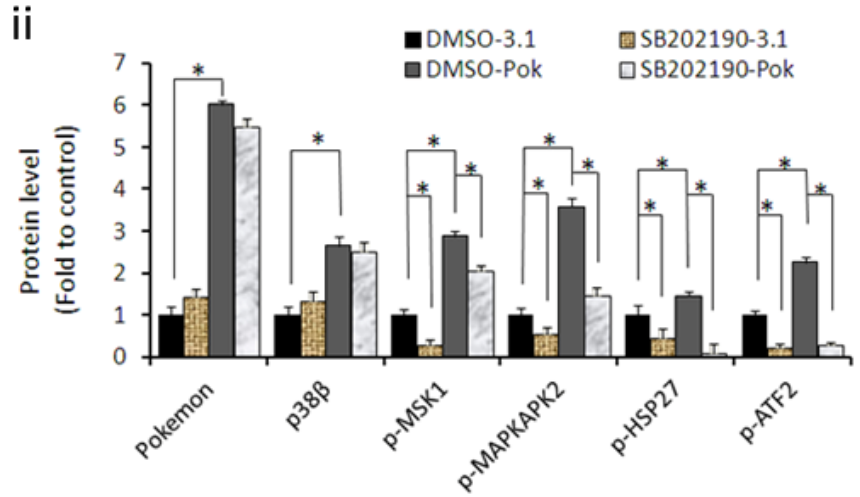

Together these data suggested that the promotion on HepG2 growth, migration and invasion by ectopic expression of Pokemon was efficiently attenuated by $25 \mu \mathrm{M}$ SB202190. Combined with the effect of Pokemon on $\mathrm{p} 38 \beta$ and the transcription factor role of Pokemon by ChIP-on-Chip assay [33], we proposed the hypothesis that the promotion of Pokemon on HepG2 cells was associated with its regulation on $\mathrm{p} 38 \beta$ expression.

\subsection{Pokemon Up-Regulates p38ß Expression in Hepatic Cells}

We performed gene-specific manipulation of Pokemon expression, focusing on the changes of p38 $\beta$ with ubiquitously existed $\mathrm{p} 38 \alpha$ as a control. We found that ectopic expression of Pokemon increased p38 $\beta$ expression at both mRNA and protein levels in HepG2 cells, but p38 $\alpha$ expression was not increased significantly (Figure 4a,b). In sharp contrast, p38 $\beta$ protein was notably reduced by si-RNA-mediated Pokemon silencing (Figure 4a). To further confirm this finding, we also up-regulated Pokemon expression in HL7702 cells and silenced Pokemon in BEL7404 cells, and we got the consistent results (Figure 4c,d). As the close association between ATF2 and p38 $\beta$ [27], the changes of downstream phosphorylated ATF2 also suggested that the ectopic expression or silence of Pokemon efficiently affected p38 $\beta$ activity, by the regulation of Pokemon on $\mathrm{p} 38 \beta$ expression. Hence, we confirmed that Pokemon only promoted p38 $\beta$ expression in hepatic cells. 
Figure 4. Pokemon up-regulates p38 $\beta$ expression in hepatic cells. Pokemon was delivered by expression plasmid pcDNA3.1(-)-Pokemon with pcDNA3.1(-) as a negative control. Pokemon silencing was triggered by si-RNA. (a) Targeted expression or silencing of Pokemon in HepG2 cells. Cells were collected at $60 \mathrm{~h}$ after transfection or silencing (Left panel). And the quantification of western blot data was displayed on the Right panel; (b) Real-time quantitative polymerase chain reaction (qPCR) at $48 \mathrm{~h}$ after transfection in HepG2 cells. Upper panel: Ectopic expression of Pokemon; lower panel: Silencing of Pokemon; (c) Ectopic expression of Pokemon in HL7702 cells. Upper panel: Western blot in which cells were lysed and total proteins were collected at 36, 48, 60 and $72 \mathrm{~h}$, respectively. Lower panel: Real-time qPCR at 48 and $60 \mathrm{~h}$ after transfection; (d) Silencing of Pokemon in BEL7404 cells. Upper panel: Western blot; lower panel: Real-time qPCR. $* p<0.05$ compared to the negative control. N.C. means negative control.

a. HepG2 cell
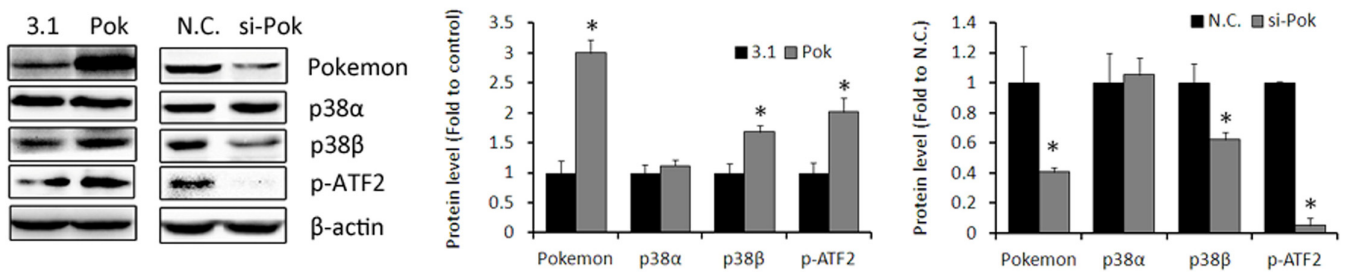

b.

c.

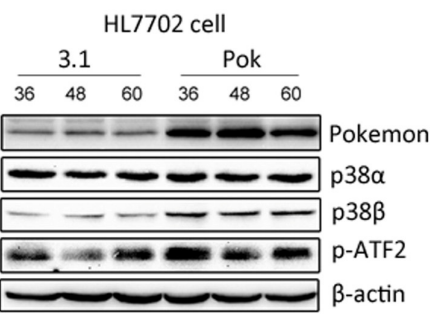

d.

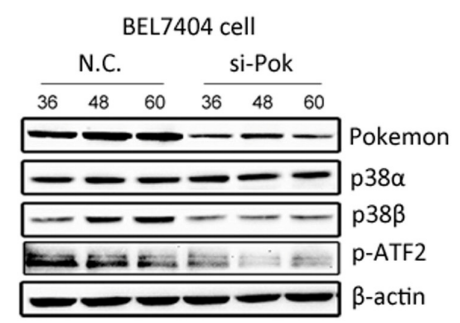
Pokemon p38 $\alpha$ p38ß ATF2
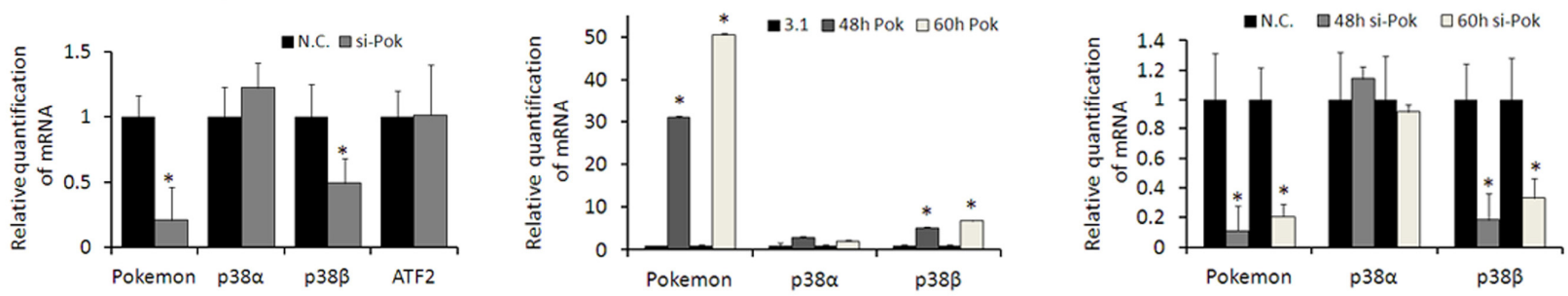

\subsection{Pokemon Promotes p38 $\beta$ Expression by Up-Regulating Its Transcription}

The effect on $\mathrm{p} 38 \beta$ mRNA level suggested that Pokemon may regulate $\mathrm{p} 38 \beta$ expression at the transcriptional level. We next examined whether Pokemon binds to the endogenous p38 $\beta$ promoter in vivo using a ChIP assay. Total lysates from HepG2 and BEL7404 cells were used as an input and positive control, while immunoprecipitates with serum IgG served as a negative control. As shown in Figure 5a, Pokemon protein bound to the $\mathrm{p} 38 \beta$ promoter approximately between -763 bp and $-415 \mathrm{bp}$ upstream of the transcriptional start site, but there was no positive signal at more distant upstream region of between $-1155 \mathrm{bp}$ and $-936 \mathrm{bp}$. To further investigate whether Pokemon transcriptionally activates $\mathrm{p} 38 \beta$ promoter, we constructed a luciferase reporter expression plasmid containing wild type p38 $\beta$ promoter sequence, and co-transfected with Pokemon expression plasmid into 293T cells to 
precede a Dual luciferase report assay. The results indicated that Pokemon indeed enhanced the promoter activity of $\mathrm{p} 38 \beta$ dose-dependently (Figure $5 b$ ).

Figure 5. Pokemon stimulates p38 promoter activity. (a) ChIP assays in HepG2 and BEL7404 cells. Protein-DNA complexes are immunoprecipitated either with anti-Pokemon antibody or anti-IgG as negative control, followed by PCR with primers specific to p38 $\beta$ promoter sequence and agarose-gel electrophoresis for visualization. Total lysates were used as the input samples and positive control; (b) Dual luciferase reporter assay. Luciferase activities were normalized to Renilla activity. Y axis stands for the relative fold changes of activity as the pcDNA3.1(-)-Pokemon plasmid increases. ${ }^{*} p<0.05$ compared to the negative control.
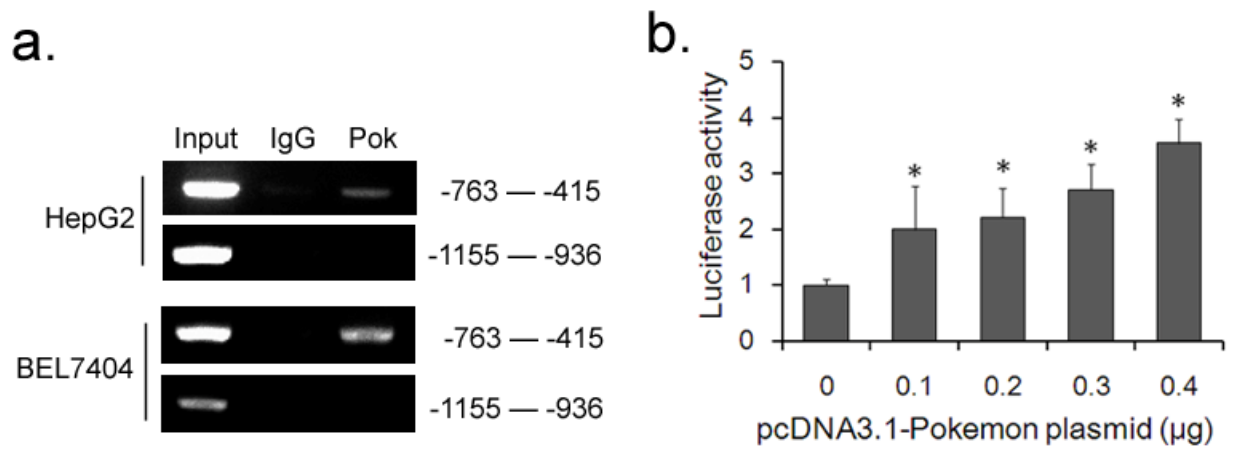

\subsection{Discussion}

As it is reported in our former ChIP-on-Chip assay, a total of 556 genes were identified as targets of Pokemon in HepG2 cells, being transcriptionally regulated [33]. Among the target genes, we find Pokemon plays a pivotal regulatory role in MAPK signaling pathways. The MAPK11 gene, which encodes $\mathrm{p} 38 \beta$, is considered as a potential direct binding target of Pokemon. In this study, we aimed to further clarify the relationship between Pokemon and p38 $\beta$, and tried to enhance the understanding of the mechanisms by which Pokemon affects cellular biologic behaviors in hepatic cells.

It was reported that Pokemon promotes the growth of ovarian cancer cells [31]. In this study, we confirmed this role of Pokemon in hepatic cells, particularly in the clonogenic growth, a more representative assay of cancer cell tumorigenicity. In the MTT cell growth assays, HepG2 cells with ectopic Pokemon expression demonstrated significant superiority in proliferation at 96 and $120 \mathrm{~h}$, which suggested that overexpressed Pokemon acted as an oncogene and rendered HepG2 cells a growth advantage at high cell density and low nutrition status. However, in HepG2 and BEL7404 cells, silencing of Pokemon did not dramatically affect cell growth. Pokemon may not be a critical oncogenic signal, and possibly other pathways play a dominant role in such state. It is noteworthy to notice that the p38 inhibitor, SB202190, greatly attenuated the cell proliferation stimulated by Pokemon, suggesting its correlation with the p38 signaling pathway.

The p38 signaling pathway is implicated in cellular migration [24]. This study demonstrated that Pokemon acted as an upstream factor of this pathway to promote cell invasion and migration, suggesting Pokemon as a potential indicator of metastatic capability of malignant hepatic cells. In addition, a combination of p38 inhibitor SB202190 with Pokemon silencing additively reduced the 
invasive capability of HepG2 cells, and a similar phenomenon has been observed in colony formation assays (Figure S3b,d). It has been reported that Pokemon promotes cell metastasis by activating MMP-1 gene in ovarian cancer [31]. Our results revealed a novel regulatory mechanism that Pokemon may regulate the metastasis of hepatic cells through a p38 $\beta$-dependent pathway.

Many reports suggest that Pokemon plays a transcription factor role in protein expression. We found that Pokemon had a promoting effect on $\mathrm{p} 38 \beta$ expression and its downstream, phosphorylated ATF2, but no significant effect on p38 $\alpha$ (Figure 4). p38 inhibitor SB202190, which specifically inhibits the activity of $\mathrm{p} 38 \beta$ and $\mathrm{p} 38 \alpha$, efficiently attenuates the levels of $\mathrm{p} 38 \beta$ and phosphorylated ATF2 triggered by Pokemon, proving the role of $\mathrm{p} 38 \alpha$ or $\mathrm{p} 38 \beta$ in this signaling. The affinity of $\mathrm{p} 38 \beta$ to ATF2 is twenty times larger than that of $\mathrm{p} 38 \alpha$ [27]. Because of the indistinctive change of ATF2 mRNA after Pokemon overexpression, we have reason to believe that the increased level of phosphorylated ATF2 is due to the up-regulation of $\mathrm{p} 38 \beta$. Nevertheless, we found only $\mathrm{p} 38 \beta$, and not p38 $\alpha$ expression, is regulated by Pokemon. According to these results, we confirmed p38 $\beta$ signaling pathway is one of the patterns by which Pokemon modulates. It is noteworthy that a live cell responds to stress in a complicated network, particularly in phosphorylation signaling transition. Therefore, in our studies, the Pokemon protein level was not proportional to the phosphorylation of the effector proteins, and these results of our study provide a potential pattern of Pokemon affecting MAPK signaling pathways.

In our study, we found that $\mathrm{p} 38 \beta$, encoded by the MAPK11 gene, is one target of Pokemon in the MAPK signaling pathways, which is consistent with the ChIP-on-Chip results [33]. In summary, our study proposed that in hepatic cells, Pokemon promotes the expression of p38 $\beta$ by directly binding to its promoter region upstream of transcription start site and thus modulates the p38 signaling; whether this is one of the mechanisms through which Pokemon acts as an oncogene and promotes cell growth, migration and invasion will require further research.

\section{Experimental Section}

\subsection{Cell Culture}

Hepatoblastoma-derived cell line HepG2, hepatocellular carcinoma cell line BEL7404, QGY7703, human immortalization hepatic cell line HL7702, Human embryo kidney cell 293T were purchased from the Chinese Academy of Sciences cell bank and cultured in RPMI-1640 or DMEM (High Glucose) medium, supplemented with $10 \%$ fetal bovine serum (FBS, Hyclone, Wilmington, DE, USA). Cells were maintained at $37{ }^{\circ} \mathrm{C}$ with $5 \% \mathrm{CO}_{2}$ atmosphere.

\subsection{Real-Time Quantitative PCR}

Pokemon expression vector pcDNA3.1(-)-Pokemon were produced in previous studies [9]. si-RNA was purchased from GenePharma, sequences are: si-Pokemon, 5'-GCU GGA CCU UGU AGA UCA ATT-3' (forward) and 5'-UUG AUC UAC AAG GUC CAG CTT-3' (reverse); negative control si-RNA (N.C.), 5'-UUC UCC GAA CGU GUC ACG UTT-3' (forward) and 5'-ACG UGA CAC GUU CGG AGA ATT-3' (reverse). Transfection assay was preceded following lipofectamine 2000 protocol (Invitrogen, Grand Island, NY, USA). Total RNA was extracted after transfection for 48 or $60 \mathrm{~h}$ using 
Trizol Reagent (Invitrogen). RNA was reversely transcribed to cDNA using PrimeScript RT Master Mix (TaKaRa, Dalian, Liaoning, China).

DNA was used for quantitative PCR analysis performed. cDNA, primers, and Master Mix (SYBR Premix Ex TaqTM, TaKaRa), were mixed as the instruction of manufacturer. Reactions were performed by a 7500 Fast Real-time PCR System (Applied Biosystems, Bedford, OH, USA). Primers were as follows: p38 $\alpha$ : 5'-TGG AAG CCT GGA CTC TAA-3' (forward) and 5'-ATC CTA TAC GGC ATA ACT G-3' (reverse); p38ß: 5'-AGA AGG TGG CGG TGA AGA-3' (forward) and 5'-CGT CCA GAA GCC CGA TGA-3' (reverse); GAPDH: 5'-GGT GGT CTC CTC TGA CTT CAA CA-3' (forward) and 5'-GTT GCT GTA GCC AAA TTC GTT GT-3' (reverse); and Pokemon: 5'-GAA GCC CTA CGA GTG CAA CAT C-3' (forward) and 5'-GTG GTT CTT CAG GTC GTA GTT GTG-3' (reverse).

\subsection{Western Blot}

Proteins were extracted from treated cells and separated in 10\%-12\% SDS-polyacrylamide gel and then transferred to a PVDF membrane (Amersham Bioscience, Piscataway, NJ, USA). The membranes were blocked in 5\% bovine serum albumin (BSA, Sangon Biotech. Shanghai, China) and then incubated overnight at $4{ }^{\circ} \mathrm{C}$ in first antibody. The membranes were washed and incubated with horseradish peroxidase-conjugated second antibody. Protein bands were visualized by an imaging system (Bio-Rad, Munich, Germany) after developed with ECL reagents (Thermo Scientific, Wilmington, DE, USA). Antibodies used are as follows: Pokemon antibody (Sigma, St. Louis, MO, USA), p38 $\alpha$ MAPK (7D6) Rabbit mAb (CST, Danvers, MA, USA), p38 $\beta$ MAPK (c28c2) Rabbit mAb (CST), Phospho-p38 MAPK Pathway Sampler Kit (CST) and Actin antibody (Beyotime, Shanghai, China).

\subsection{Dual Luciferase Reporter Assay}

We constructed wide-type promoter plasmid pGL4.10-p38 $\beta$, empty pGL4.10 was used as a negative control. Primers for promoters are 5'-CCC GGT ACC GCT GGC TGT TTT AAT TTG G -3' (forward) and 5'-TAA CTC GAG AGC TCC TGC CGG TAG AAG-3' (reverse), with KpnI and XhoI restriction enzyme cut sites.

This assay was preceded in 293T cells because of their high transfection efficiency. After transfection for $36 \mathrm{~h}$, cells were dissolved with passive lysis buffer $(1 \times)$. Luciferase activity was measured using a Dual luciferase reporter assay kit (Promega, Fitchburg, WI, USA).

\subsection{Chromatin Immunoprepcipitation (ChIP)}

ChIP assay was preceded according to ChIP-IT ${ }^{\circledR}$ express enzymatic magnetic chromatin immunoprecipitation kit \& enzymatic shearing kit protocols. DNA fragments were used as templates to preced PCR. Primers are as follows: Between $-763 \mathrm{bp}$ and $-415 \mathrm{bp}$ : 5'-TGG AGG GGG TCG CCC AGC CGC GAA G-3' (forward) and 5'-TGC CCA GAA CCT TTC CTC CT-3' (reverse); between -1155 bp and -936 bp: 5'-GGA CTG AGA CCC GTT CCT TCG-3' (forward) and 5'-TGG TAG AGC CGT GGT GGG AG-3' (reverse). Antibodies used are as follows: Anti-Pokemon antibody (Abcam, Cambridge, UK) and Goat Control IgG antibody (Abcam). 


\subsection{MTT Assay}

Cells were seeded at a density of $3 \times 10^{3} / 100 \mu \mathrm{L}$ medium in 96-well plate and treated with the SB202190 (Merck Millipore, Darmstadt, Germany) at different time points, ranging from 24 to $120 \mathrm{~h}$. Cells were incubated with MTT $(5 \mathrm{mg} / \mathrm{mL})$ for $4 \mathrm{~h}$ and formazan precipitate was dissolved in $100 \mu \mathrm{L}$ DMSO and the absorbance at $595 \mathrm{~nm}$ was measured by Multimode Detector DTX880 (Beckman Coulter, Atlanta, GA, USA).

\subsection{Colony Formation Assay}

HepG2 cells at the exponential phase were plated into 24-well plate (200-300 cells/well) and allowed to adhere for $12 \mathrm{~h}$ before treatment. Then the culture medium was substituted with fresh medium containing SB202190 and incubated for 14 days. Cells were then counted and photographed after rinsed with PBS and stained with $1.0 \%$ crystal violet.

\subsection{Invasion and Migration Assays}

The invasion ability of cells was measured by the Boyden chamber invasion assay. Matrigel (Sigma) was diluted at the ratio of 1:1 with pre-cooled culture medium and added on the upper chamber of the $8 \mu \mathrm{m}$ pore polycarbonate filter (Merck Millipore). The medium containing 10\% FBS was added in the lower chamber, and the treated cells were re-suspended in serum-free medium and plated on the upper chamber. After $24 \mathrm{~h}$ incubation at $37{ }^{\circ} \mathrm{C}$, the cells in the upper surface were carefully removed with a cotton swab and cells invaded across the matrigel were stained with crystal violet, counted and photographed.

The migration assay was preceded similarly as the invasion assay described above, except the upper chamber of polycarbonate filters were not coated with matrigel.

\subsection{Statistical Analysis}

All Data were presented as mean \pm S.E.M. from experiments done in triplicate. Statistical analyses were performed using Student's $t$-test. The difference was considered statistically significant when the $p$ value was $<0.05$.

\section{Conclusions}

Pokemon is considered a pivotal transcription factor, involved in multiple biological processes [12,13] and localized upstream of several oncogenes [17]. In this study, we confirmed the promoting effect of Pokemon on cell growth, migration and invasion in hepatic cells. Through the attenuation of the promotion by SB202190, we uncovered a new mechanism that Pokemon is involved in. Pokemon transcriptionally up-regulates the expression of $\mathrm{p} 38 \beta$ and consequently stimulates p38 signaling. 


\section{Acknowledgments}

We thank the financial support from the Ministry of Science and Technology of China (2012ZX09506001-010, 2012CB722605, 2012AA020305 and 2011DFA30620) and the National Natural Science Foundation of China (21172129).

\section{Conflict of Interest}

The authors declare no conflict of interest.

\section{References}

1. Pessler, F.; Pendergrast, P.S.; Hernandez, N. Purification and characterization of FBI-1, a cellular factor that binds to the human immunodeficiency virus type 1 inducer of short transcripts. Mol. Cell Biol. 1997, 17, 3786-3798.

2. Maeda, T.; Ito, K.; Merghoub, T.; Poliseno, L.; Hobbs, R.M.; Wang, G.; Dong, L.; Maeda, M.; Dore, L.C.; Zelent, A.; et al. LRF is an essential downstream target of GATA1 in erythroid development and regulates BIM-dependent apoptosis. Dev. Cell 2009, 17, 527-540.

3. Maeda, T.; Merghoub, T.; Hobbs, R.M.; Dong, L.; Maeda, M.; Zakrzewski, J.; van den Brink, M.R.; Zelent, A.; Shigematsu, H.; Akashi, K.; et al. Regulation of B versus T lymphoid lineage fate decision by the proto-oncogene LRF. Science 2007, 316, 860-866.

4. Collins, T.; Stone, J.R.; Williams, A.J. All in the family: The BTB/POZ, KRAB, and SCAN domains. Mol. Cell Biol. 2001, 21, 3609-3615.

5. Maeda, T.; Hobbs, R.M.; Merghoub, T.; Guernah, I.; Zelent, A.; Cordon-Cardo, C.; Teruya-Feldstein, J.; Pandolfi, P.P. Role of the proto-oncogene Pokemon in cellular transformation and ARF repression. Nature 2005, 433, 278-285.

6. Weinberg, R.A. The cat and mouse games that genes, viruses, and cells play. Cell 1997, 88, 573-575.

7. Aggarwal, H.; Aggarwal, A.; Hunter, W.J.; Yohannes, P.; Khan, A.U.; Agrawal, D.K. Expression of leukemia/lymphoma related factor (LRF/Pokemon) in human benign prostate hyperplasia and prostate cancer. Exp. Mol. Pathol. 2011, 90, 226-230.

8. Aggarwal, A.; Hunter, W.J.; Aggarwal, H.; Silva, E.D.; Davey, M.S.; Murphy, R.F.; Agrawal, D.K. Expression of leukemia/lymphoma-related factor (LRF/POKEMON) in human breast carcinoma and other cancers. Exp. Mol. Pathol. 2010, 89, 140-148.

9. Zu, X.; Ma, J.; Liu, H.; Liu, F.; Tan, C.; Yu, L.; Wang, J.; Xie, Z.; Cao, D.; Jiang, Y. Pro-oncogene Pokemon promotes breast cancer progression by upregulating survivin expression. Breast Cancer Res. 2011, 13, doi:10.1186/bcr2843.

10. Rovin, R.A.; Winn, R. Pokemon expression in malignant glioma: An application of bioinformatics methods. Neurosurg. Focus 2005, 19, E8.

11. Nakayama, K.; Nakayama, N.; Wang, T.L.; Shih, I.M. NAC-1 controls cell growth and survival by repressing transcription of Gadd45GIP1, a candidate tumor suppressor. Cancer Res. 2007, $67,8058-8064$. 
12. Choi, W.I.; Jeon, B.N.; Park, H.; Yoo, J.Y.; Kim, Y.S.; Koh, D.I.; Kim, M.H.; Kim, Y.R.; Lee, C.E.; Kim, K.S.; et al. Proto-oncogene FBI-1 (Pokemon) and SREBP-1 synergistically activate transcription of fatty-acid synthase gene (FASN). J. Biol. Chem. 2008, 283, 29341-29354.

13. Choi, W.I.; Jeon, B.N.; Yun, C.O.; Kim, P.H.; Kim, S.E.; Choi, K.Y.; Kim, S.H.; Hur, M.W. Proto-oncogene FBI-1 represses transcription of $p 21$ CIP1 by inhibition of transcription activation by p53 and Sp1. J. Biol. Chem. 2009, 284, 12633-12644.

14. Jeon, B.N.; Yoo, J.Y.; Choi, W.I.; Lee, C.E.; Yoon, H.G.; Hur, M.W. Proto-oncogene FBI-1 (Pokemon/ZBTB7A) represses transcription of the tumor suppressor $R b$ gene via binding competition with Sp1 and recruitment of co-repressors. J. Biol. Chem. 2008, 283, 33199-33210.

15. Zhang, N.N.; Sun, Q.S.; Chen, Z.; Liu, F.; Jiang, Y.Y. Homeostatic regulatory role of Pokemon in NF- $\kappa \mathrm{B}$ signaling: Stimulating both $\mathrm{p} 65$ and $\mathrm{I} \kappa \mathrm{B} \alpha$ expression in human hepatocellular carcinoma cells. Mol. Cell Biochem. 2012, 372, 57-64.

16. Lee, D.K.; Kang, J.E.; Park, H.J.; Kim, M.H.; Yim, T.H.; Kim, J.M.; Heo, M.K.; Kim, K.Y.; Kwon, H.J.; Hur, M.W. FBI-1 enhances transcription of the nuclear factor- $\mathrm{KB}$

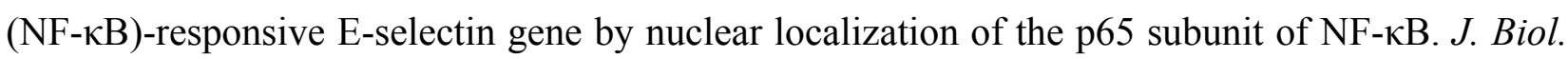
Chem. 2005, 280, 27783-27791.

17. Liu, K.; Liu, F.; Zhang, N.N., Liu, S.Y.; Jiang, Y.Y. Pokemon Silencing leads to Bim-mediated Anoikis of Human Hepatoma Cell QGY7703. Int. J. Mol. Sci. 2012, 13, 5818-5831.

18. Nebreda, A.R.; Porras, A. p38 MAP kinases: Beyond the stress response. Trends Biochem. Sci. 2000, 25, 257-260.

19. Ono, K.; Han, J. The p38 signal transduction pathway: Activation and function. Cell Signal. 2000, $12,1-13$.

20. Kyriakis, J.M.; Avruch, J. Mammalian mitogen-activated protein kinase signal transduction pathways activated by stress and inflammation. Physiol. Rev. 2001, 81, 807-869.

21. Cuenda, A.; Rousseau, S. p38 MAP-kinases pathway regulation, function and role in human diseases. Biochem. Biophys. Acta 2007, 1773, 1358-1375.

22. Lee, J.C.; Laydon, J.T.; McDonnell, P.C.; Gallagher, T.F.; Kumar, S.; Green, D.; McNulty, D.; Blumenthal, M.J.; Heys, J.R.; Landvatter, S.W.; et al. A protein kinase involved in the regulation of inflammatory cytokine biosynthesis. Nature 1994, 372, 739-746.

23. Wagner, E.F.; Nebreda, A.R. Signal integration by JNK and p38 MAPK pathways in cancer development. Nat. Rev. Cancer 2009, 9, 537-549.

24. Xu, L.; Chen, S.; Bergan, R.C. MAPKAPK2 and HSP27 are downstream effectors of p38 MAP kinase-mediated matrix metalloproteinase type 2 activation and cell invasion in human prostate cancer. Oncogene 2006, 25, 2987-2998.

25. Deak, M.; Clifton, A.D.; Lucocq, L.M.; Alessi, D.R. Mitogen- and stress-activated protein kinase-1 (MSK1) is directly activated by MAPK and SAPK2/p38, and may mediate activation of CREB. EMBO J. 1998, 17, 4426-4441.

26. Patel, S.B.; Cameron, P.M; O’Keefe, S.J.; Frantz-Wattley, B.; Thompson, J.; O’Neill, E.A.; Tennis, T.; Liu, L.; Becker, J.W.; Scapin, G. The three-dimensional structure of MAP kinase p38 $\beta$ : Different features of the ATP-binding site in $\mathrm{p} 38 \beta$ compared with $\mathrm{p} 38 \alpha$. Acta Crystallogr. D 2009, 65, 777-785. 
27. Jiang, Y.; Chen, C.; Li, Z.; Guo, W.; Gegner, J.A.; Lin, S.; Han, J. Characterization of the structure and function of a new mitogen-activated protein kinase (p38ß). J. Biol. Chem. 1996, 271, 17920-17926.

28. Guo, Y.L.; Kang, B.; Han, J.; Williamson, J.R. p38 $\beta$ MAP kinase protects rat mesangial cells from TNF- $\alpha$-induced apoptosis. J. Cell Biochem. 2001, 82, 556-565.

29. Kim, H.P.; Wang, X.; Nakao, A.; Kim, S.I.; Murase, N.; Choi, M.E.; Ryter, S.W.; Choi, A.M. Caveolin-1 expression by means of $\mathrm{p} 38 \beta$ mitogen-activated protein kinase mediates the antiproliferative effect of carbon monoxide. Proc. Natl. Acad. Sci. USA 2005, 102, 11319-11324.

30. Kalender, A.; Selvaraj, A.; Thomas, G. A matter of energy stress: p38 $\beta$ meets mTORC1. Cell Res. 2011, 21, 859-861.

31. Jiang, L.; Siu, M.K.; Wong, O.G.; Tam, K.F.; Lam, E.W.; Ngan, H.Y.; Le, X.F.; Wong, E.S.; Chan, H.Y.; Cheung, A.N. Overexpression of proto-oncogene FBI-1 activates membrane type 1-matrix metalloproteinase in association with adverse outcome in ovarian cancers. Mol. Cancer 2010, 9, doi:10.1186/1476-4598-9-318.

32. Nemoto, S.; Xiang, J.; Huang, S.; Lin, A. Induction of apoptosis by SB202190 through inhibition of p38 $\beta$ mitogen-activated protein kinase. J. Biol. Chem. 1998, 273, 16415-16420.

33. Zu, X.; Yu, L.L.; Sun, Y.; Tian, J.; Liu, F.; Sun, Q.S.; He, S.N.; Sun, G.; Luo, W.S.; Jiang, Y.Y. Global mapping of ZBTB7A transcription factor binding sites in HepG2 cells. Cell Mol. Biol. Lett. 2010, 15, 260-271.

(C) 2013 by the authors; licensee MDPI, Basel, Switzerland. This article is an open access article distributed under the terms and conditions of the Creative Commons Attribution license (http://creativecommons.org/licenses/by/3.0/). 